\title{
Relationship between Nutrition (REAP), Exercise (VSAQ), and Stress on Premenstrual Syndrome Severity (PSST): Correlation, Cross-Section, Purposive Sample of 75 Females Ages 18-55
}

Walton LM ${ }^{1 *}$, Machamer $\mathrm{L}^{2}$, Asumbrado RC ${ }^{2}$, Behrens MA ${ }^{2}$

${ }^{1}$ Department of Neuroscience, University of Sharjah, Johns Hopkins University Bloomberg School of Public Health, United Arab Emirates ${ }^{2}$ Department of Neuroscience, Andrews University, Berrien Springs, United Arab Emirates

\begin{abstract}
The purpose of this study was to determine if specific factors of exercise (type, intensity, frequency, and duration), nutritional intake, or perceived stress were able to predict the PMS symptom severity. This was a prospective, correlation, cross-sectional study, purposive sample of healthy females $(\mathrm{N}=75)$, ages $18-55$ years of age. This study was approved by Andrews University IRB and voluntary informed consent was given prior to data collection. Factors of exercise were examined using the Veterans Specific Activity Questionnaire (VSAQ). Nutrition was examined using the Rapid Eating Assessment for Patients (REAP). PMS symptoms were measured using the Premenstrual Symptoms Screening Tool (PSST) on 75 women $(\mathrm{N}=75)$ (Mean=22.7 years). Multiple linear regression was used to analyze predictive ability of exercise intensity and nutrition on the severity of PMS symptom severity. No significant predictive relationship between VSAQ (exercise intensity) or nutritional intake scores and PMS severity was found. However, a significant relationship between perceived stress and PMS symptoms was reported for the sample $(r=0.324, n=75, p<0.005)$. In conclusion, perceived stress levels were found to have a direct, significant correlation to PMS symptom severity. However, factors related to exercise and nutrition were not significant predictors of PMS symptom severity according to this research study. Future studies should focus on prospective, experimental studies to examine specific exercise and nutritional prescription, with sampling of cortisol levels during the menstrual cycle
\end{abstract}

Keywords: Nutrition; Exercise; PSST; Stress; Women's health

\section{Introduction}

Premenstrual Syndrome (PMS) affects $75-90 \%$ of menstruating women [1,2]. PMS can lead to dysfunction in social, mental and physical wellbeing directly impacting a woman's quality of life [1-5]. The multitude of symptoms reported, including but not limited to dysphoria, mood swings, fatigue, headaches, and irritability, add difficulty for diagnosis and treatment of PMS $[1,3,6]$. Furthermore, leading organizations such as the World Health Organization (WHO), National Institute of Mental Health (NIMH) and American College of Obstetrics and Gynecology (Bulletin), provide different guidelines for diagnosing PMS [2,7].

Factors related to PMS are often considered outside rehabilitation medicine and inaccurately labeled as psychological, meanwhile avoiding physiological implications of cyclic pain in rehabilitation. The research has focused on the influence of sleep cycles, hormones, thyroid indices, changes in circadian rhythm and timing deviations in excretion of melatonin, cortisol, thyroid-stimulating hormone, as well as prolactin factor contribution to premenstrual syndrome severity [8,9]. Multiple studies also suggest symptoms may be related to epigenetic response to normal changes in hormone levels $[4,9]$. Numerous interventions have been studied to find a method to minimize the negative effects of PMS for women. However, few studies have considered the effects of specific factors related to exercise, nutrition, and perceived stress on PMS severity.

American College of Obstetricians and Gynecologists (ACOG) recommends exercise as a means to reduce the symptoms of PMS (fatigue, bloating, pain) and to increase the positive well-being for women who suffer from PMS $[1,10]$. Studies suggest exercise directly affects hormonal controls, via the endocrine system, and may play a large role in regulating related PMS symptoms [11]. One study examined hormonal and lactate responses to high intensity iso-kinetic exercise in endurance trained and sedentary subjects to see if physical training levels decreased efficiency of hypothalamic pituitary adrenal (HPA) axis. No difference in activation or recovery values occurred between trained and sedentary subjects, reinforcing the idea that similar physiological responses occur regardless of fitness level during exercise. Results suggest adrenocortical activation responds to exercise and is not dependent on an individual's fitness level. This study provides evidence of exercise direct benefit for women, independent of fitness level, and ability to immediately and positively impact the endocrine system [2].

Although research supports exercise for reducing PMS symptoms, intensity and amount of exercise is not standardized and vague. A study of subjects $(\mathrm{N}=61)$ concluded significant decrease in menstrual symptoms for moderate and high intensity groups. Both groups significantly improved their high-density lipoprotein (HDL), triglycerides (TGL), VO2 max, forced vital capacity (FVC) and maximum voluntary ventilation (MVV) [2]. Another study $(\mathrm{N}=90)$ found that aerobic exercise, with either moderate or high intensity exercise, effectively reduced PMS symptoms. There was reduced

*Corresponding author: Lori Maria Walton, Department of Neuroscience, University of Sharjah, Johns Hopkins University Bloomberg School of Public Health, United Arab Emirates, Tel: +13159305144; E-mail: loriptdoc@gmail.com/ walton8@jhu.edu

Received: March 15, 2018; Accepted: May 16, 2018; Published: May 21, 2018

Citation: Walton LM, Machamer L, Asumbrado RC, Behrens MA (2018) Relationship between Nutrition (REAP), Exercise (VSAQ), and Stress on Premenstrual Syndrome Severity (PSST): Correlation, Cross-Section, Purposive Sample of 75 Females Ages 18-55. Physiother Rehabil 3: 158. doi: 10.4172/2573-0312.1000158

Copyright: (c) 2018 Walton LM, et al. This is an open-access article distributed under the terms of the Creative Commons Attribution License, which permits unrestricted use, distribution, and reproduction in any medium, provided the original author and source are credited. 
Citation: Walton LM, Machamer L, Asumbrado RC, Behrens MA (2018) Relationship between Nutrition (REAP), Exercise (VSAQ), and Stress on Premenstrual Syndrome Severity (PSST): Correlation, Cross-Section, Purposive Sample of 75 Females Ages 18-55. Physiother Rehabil 3: 158. doi: 10.4172/2573-0312.1000158

depressive mood, water retention and discomfort with exercise of a 10-minute warming, 30-minute jog and 10-minute cool down, three times a week for 3 months [12]. Further research is needed to clarify the effects of varying intensity on symptoms.

Research supports nutrition intake for reducing PMS symptoms, but as with exercise specific amount is not standardized and vague. Bosarge [13] suggests lifestyle changes for treating symptoms of PMS, including vitamin B, calcium supplements and limiting sodium, caffeine and alcohol intake. Speculation exists for Magnesium, 400 $\mathrm{mg}$, helping with metabolizing fatty acids and reducing fluid retention and mood swings. Vitamin $\mathrm{E}$ is suggested for treating anxiety, tension breast tenderness and headaches during all parts of the menstrual cycle [13]. Both Wakeman and Chocano-Bedoya et al. [3,14] suggest using vitamin B to decrease symptoms of PMS by increasing serotonin and dopamine production. Case-control study $(\mathrm{N}=1057)$ found increased levels of thiamine \& riboflavin from food sources, to be inversely related to incidence of PMS [14]. These data are consistent with research supporting specific nutrition intake and decreased PMS symptoms $[3,14,15]$.

The prostaglandin theory suggests intake of fatty acids as a treatment method for reducing PMS symptoms [3,14]. Sohrabi et al. [16] presents significant research on the positive effects of omega- 3 fatty acids on the psychiatric symptoms of PMS. Rocha Filho et al. [15] studied the effects of a medicine infused with essential fatty acids and found reduced symptoms of PMS. Baseline level scores revealed that symptoms were significantly higher during the luteal phase in all groups. By the third month of treatment, groups receiving the medication had significant change in PMS symptoms determined by prospective record of impact and severity of menstruation (PRISM). Suggesting decreased symptoms of PMS due to the positive effects of fatty acids (Physicians, Friedrich). Wakeman [3] found anti-inflammatory effect of Omega 3 fatty acids that decreased the amount of inflammatory response, hence symptoms. Omega 3 fatty acids, present in krill oil, Eicosapentaenoic acid (EPA), and docosahexaenoic acid all showed statistically significant difference for the reduction of global PMS.

The purpose of this study was to examine the predictive ability for factors of exercise utilizing the Veterans Specific Activity Questionnaire (VSAQ), perceived stress on a Visual Analogue Scale (VAS Stress Scale), and nutritional intake per the Rapid Eating Assessment for Patients Survey (REAP) to determine PMS symptom severity. Our research questions include: (1) Are exercise intensity, duration, and/ or nutritional intake able to predict PMS symptoms in healthy females between the ages of 18-55? (2) Does a diet that includes calcium, vitamin $B, C, D$, magnesium and vitamin $E$ contribute to reduction in PMS symptom severity? (3) Does stress level, as reported on the VAS Stress Scale relate to PMS symptom severity?

\section{Methods}

\section{Participants}

Females $(\mathrm{N}=75)$, between the ages of $18-55$ years old, were surveyed after obtaining IRB Approval from AU Ethics Board and voluntary written consent was provided. Participants completed the following questionnaires: General Health Questionnaire, PSST, VSAQ and REAP.

\section{Validity \& Reliability of Measurement Tools}

The measurement of PMS severity was determined by completion of the PSST. Steiner et al. [17] developed the PSST to effectively and efficiently identify PMS. The tool includes a 4-point rating system: not at all, mild, moderate, and severe scale on how PMS affect participants. This research states that this tool is in line with the other studies that report prevalence of PMS [17].

The measurement of nutritional intake was determined by completion of the REAP. It is a shortened version of a food frequency questionnaire that is designed as a short dietary assessment $[18,19]$. It includes the categories in the Food Pyramid. A series of 27 questions about frequency covered four categories: 1) usually/often, 2) sometimes, 3) rarely/never, and 4) does not apply to me $[19,20]$. The test-retest reliability is excellent $(c=0.86, p<0.0001)$ and correlated with the Healthy Eating Index score $[19,20]$.

In order to measure the intensity of exercise in metabolic equivalents (METs), Veterans Specific Activity Questionnaire (VSAQ) was created. The findings stated that VSAQ, correlated with age, was more significant (multiple R value 0.72 ) then estimating METS by using heart rate, forced expiratory volume (done for 1 second), age, and BMI $(\mathrm{R}=0.58)[21]$.

\section{Measures and Procedures}

Participants were recruited at University Campuses and Health Clubs in the region. Institutional Review Board (IRB) granted approval for the study prior to data collection. Cross-sectional data collection ensued over a 4 month period. A total of 10 collection sessions were offered for all locations.

Participants who volunteered to participate in the study and met the inclusion criteria and signed the informed consent prior to completion of any surveys. Inclusion criteria was as follows: monthly menses for the last three months and did not report any of the following diagnosis: thyroid disease, pregnancy, cancer, currently taking hormonal replacement for pre-menopause or menopause, no monthly menses for at least two months in a row, currently taking routine medications (except for vitamins or over the counter medications), been diagnosed with PMDD, or any psychological disorder such as depression or bipolar disorder, diabetes and any diagnosed systemic disease. Each participant then filled out four questionnaires: general health, PSST, REAP, and VSAQ. Cardboard dividers were used to facilitate privacy and confidentiality between subjects.

Completed surveys were scored and data was recorded in SPSS. Data entry was completed by one research group member and independently checked by two other research group members for agreement. The PSST data included severity of PMS determined by diagnosis and total score. The VSAQ data included perceived exercise level reported in METs. The REAP data included frequencies on nutritional intake and included a total score and dietitian referral. The age ranges of the women were described using means and standard deviations. SPSS version 21.0 was utilized for data input and analysis. A Linear Regression was used to analyze prediction relationships between severity of PMS and exercise type, intensity, perceived stress levels and nutritional intake. A prediction formula was calculated for each variable in relationship to the outcome on the PSST.

\section{Results}

\section{Description of the sample}

Women $(\mathrm{N}=75)$ between the ages of 18 and 36 with a mean age of 22.72 and a standard deviation of 3.45 years completed this study. Figure 1 illustrates that $52.0 \%(\mathrm{~N}=39 / 75)$ were between the ages of 18 $22,41.3 \%(\mathrm{~N}=31 / 75)$ were $23-27$ years old, $4.0 \%(\mathrm{~N}=3 / 75)$ were $28-32$ years old and $2.7 \%(\mathrm{~N}=2 / 75)$ were 33-37 years old. 
Citation: Walton LM, Machamer L, Asumbrado RC, Behrens MA (2018) Relationship between Nutrition (REAP), Exercise (VSAQ), and Stress on Premenstrual Syndrome Severity (PSST): Correlation, Cross-Section, Purposive Sample of 75 Females Ages 18-55. Physiother Rehabil 3: 158. doi: 10.4172/2573-0312.1000158

Page 3 of 6

The majority were Caucasian, 33.3\%, $(\mathrm{N}=25 / 75)$ and Asian Pacific Islander, 22.7\%, (N=17/75). Figure 2 illustrates ethnic background of participants and includes the frequency and percent.

The mean BMI score for total subjects was 23.37 with a standard deviation of 4.32 and a range of 17.3-41.34. Figure 3 illustrates that $61.3 \%(\mathrm{~N}=46 / 75)$ of participants reported a BMI between $18-24,28.0 \%$ $(\mathrm{N}=21)$ reported a $\mathrm{BMI}$ of $25-29,5.3 \%(\mathrm{~N}=4)$ reported a $\mathrm{BMI}<18$ and $4.0 \%(\mathrm{~N}=3 / 75)$ reported BMI of 30-39. Visual of the BMI's for the 75 women is illustrated in Figure 3.

\section{Description of the data}

All 75 women completed the PSST, VSAQ, REAP and a general questionnaire. The PSST was scored to determine classification of moderate/severe PMS, PMDD or no diagnosis (Figure 4). The majority, $80.0 \%,(\mathrm{~N}=60 / 75)$ of the women did not meet criteria for PMS or PMDD diagnosis. PSST total scores were determined to review overall symptom rating instead of diagnosis. A 4-point scale was used to score the survey. Answers of "not at all" received 1 point and answers of "severe" received 4 points. Maximum score was 76 and minimum score was 19 or less. Figure 5 illustrates that only $1.3 \%(\mathrm{~N}=1 / 75)$ of women surveyed report no symptoms associated with their menstruation.

The VSAQ reported perceived level of fitness in MET. The lowest MET the subject underlined was reported as participant's MET level (Figure 6). The mean VSAQ was 8.08 METs with SD of 2.81 METs. Figure 6 illustrates the frequency of reported MET levels. 88\% $(\mathrm{N}=66 / 75)$ of women reported a MET level greater 7 , vigorous activity. REAP was also reported as a total score. Again, a 4- point scale was used for scoring, 1 point for an answer of "Does not apply to me" and

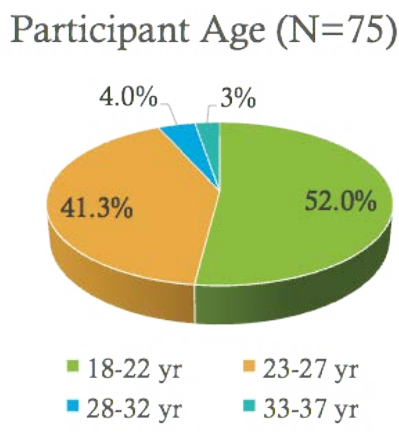

Figure 1: Frequency of age group for the 75 women surveyed.

\section{Ethnicity $(\mathrm{N}=75)$}
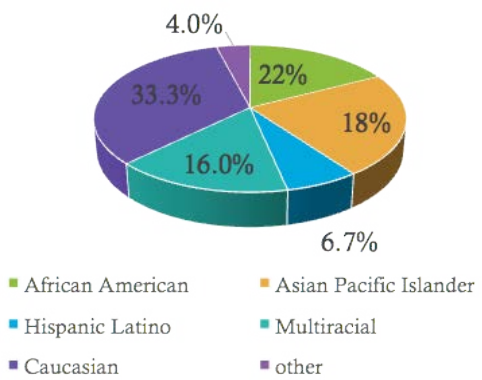

Figure 2: Ethnicity frequency and percent for the 75 women surveyed.

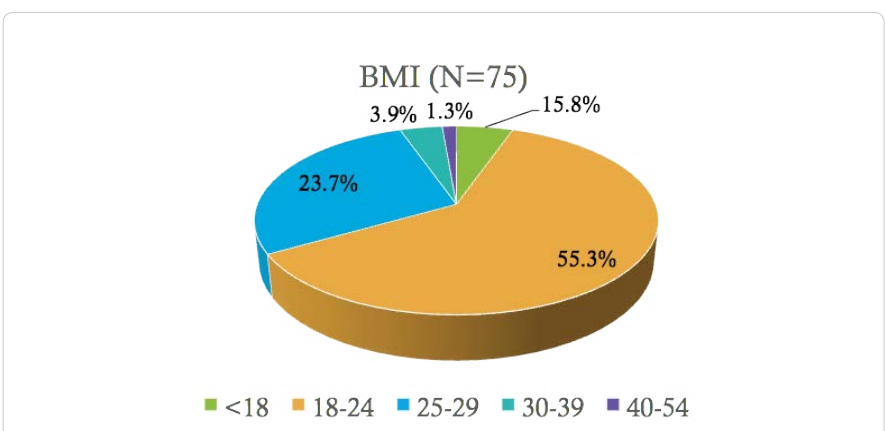

Figure 3: Frequency of BMI and percent of BMI groups for the 75 women surveyed.

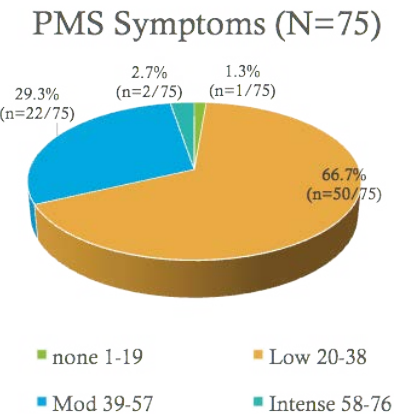

Figure 4: Frequency, in percent, of PMS symptoms.

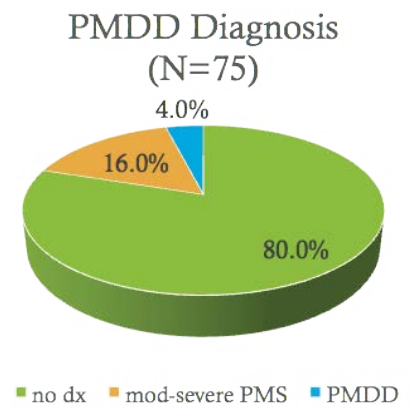

Figure 5: Frequency and percent of symptoms groups as determined by PSST total scores.

4 points for an answer of "usually/often." The mean score was 79.63 and the SD was 10.04 . Of the 75 women, $64.0 \%$ (48/75) scored between 76-100 total points, less healthy eating habits. Figure 7 includes a summary for all REAP total score groups. The REAP was also scored by total number of questioned answered "Usually/Often." A score of 5 or answered "Usually/Often" was evidence for dietitian referral. Figure 8 shows that $41 / 75$ women did not meet requirement for referral and that $34 / 75$ women did.

Figure 9 illustrates the mean stress Level, VSAQ, REAP total and REAP referral for both "no $\mathrm{dx}$ " and "PMS/PMDD" groups as determined by PSST. The mean stress level reported for "no $\mathrm{dx}$ " was 1.90; with a diagnosis the mean was 2.40. The mean VSAQ reported for "no dx" was 9.18; with a "diagnosis" of PMS/PMDD score the mean was 
Citation: Walton LM, Machamer L, Asumbrado RC, Behrens MA (2018) Relationship between Nutrition (REAP), Exercise (VSAQ), and Stress on Premenstrual Syndrome Severity (PSST): Correlation, Cross-Section, Purposive Sample of 75 Females Ages 18-55. Physiother Rehabil 3: 158. doi: 10.4172/2573-0312.1000158

Page 4 of 6

\section{Exercise Intensity}

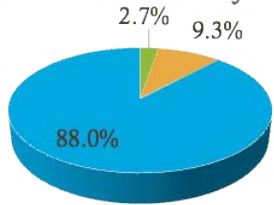

$\|$ Low (1-2) $=\operatorname{Mod}(3-6) \quad$ "Vigorous (>7)

Figure 6: Frequency and percent of MET levels reported by VSAQ.

REAP total $(\mathrm{N}=75)$

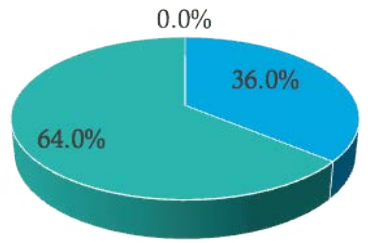

$=1.0-25=26-50=51-75=76-100$

Figure 7: Frequency, in percent, of REAP total scores.

\section{Frequency for}

Dietitian Referral

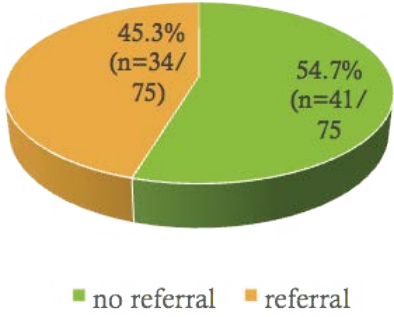

Figure 8: Frequency for dietitian referral as determined by REAP

8.67. The mean for REAP total for "no dx" was 79.82; with a "diagnosis" of PMS/PMDD score the mean was 77.53. Results are also summarized in Table 1.

Stress level was self-reported on the general health questionnaire as low, moderate or high. Figure 10 shows each stress level and the number of women with "no diagnosis" and "PMS/PMDD." Out of the 60/75 women who were not diagnosed by the PSST with PMS/PMDD, 14/60 reported low stress, 38/60 reported moderate stress and 8/60 reported high stress levels. Of the 15/75 who were diagnosed by the PSST with PMS/PMDD, 0/15 reported low stress, 9/15 reported moderate stress and 6/15 reported high stress levels.

Frequencies in age groups for women with and without diagnosis are shown in Figure 11. In the 18-22 years old age group 30/60 had no diagnosis of PMS/PMDD and 9/15 did. Age group 23-27 years old 26/60 no diagnosis of PMS/PMDD and 5/15 did. Age group 28-32 years old 2/60 no diagnosis of PMS/PMDD and 1/15 did. Age group 33-37 years old 2/60 no diagnosis of PMS/PMDD and 0/15 did.

\section{Hypothesis testing}

The purpose of the study was to determine if a predictive relationship existed between exercise intensity, nutrition and the

\begin{tabular}{|c|c|c|c|}
\hline \multirow{2}{*}{ Category } & stress level & REAP total & VSAQ \\
\cline { 2 - 4 } & Mean \pm SD & Mean \pm SD & Mean \pm SD \\
\hline No symptoms & $2.00 \pm 0.00$ & $96.00 \pm 0.00$ & $10.00 \pm 0.00$ \\
\hline Mild symptoms & $1.92 \pm 0.600$ & $78.92 \pm 9.903$ & $9.14 \pm 3.031$ \\
\hline Moderate symptoms & $2.14 \pm 0.640$ & $79.77 \pm 10.447$ & $8.95 \pm 2.591$ \\
\hline Severe symptoms & $2.5 \pm 0.707$ & $77.5 \pm 7.778$ & $8.5 \pm 2.121$ \\
\hline
\end{tabular}

Table 1: Mean stress level, VSAQ, REAP total for no, mild, moderate and severe symptoms as determined by PSST.

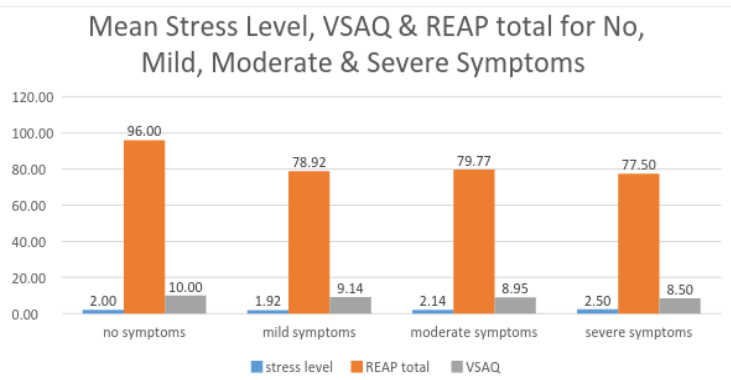

Figure 9: Mean Stress Level, VSAQ, REAP total for no, mild, moderate and severe symptoms as determined by PSST.

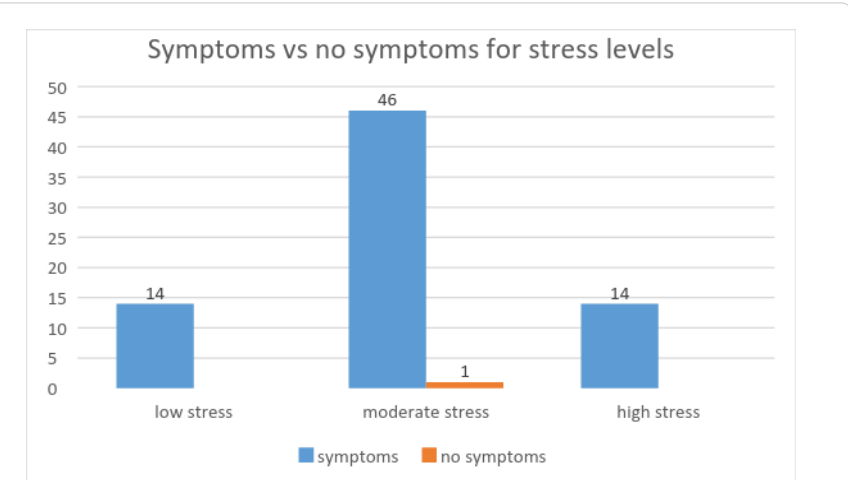

Figure 10: Stress level frequencies and percent's between PSST diagnosis groups.

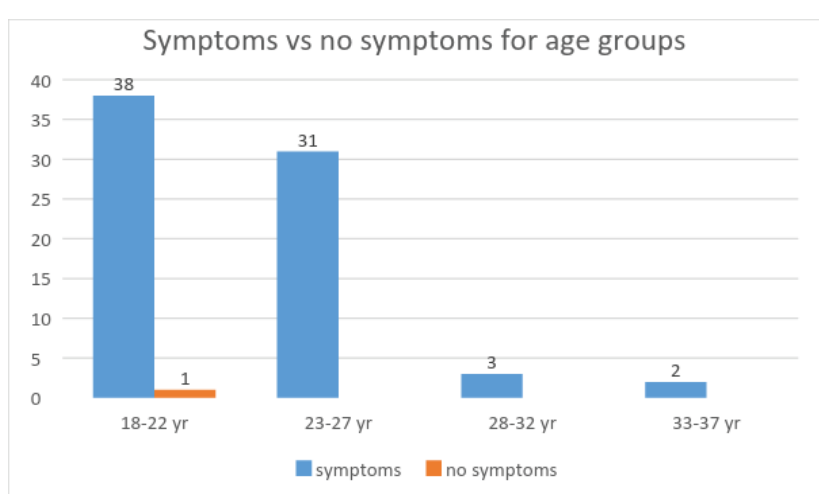

Figure 11: Symptoms vs no symptoms compared between age group. 
Citation: Walton LM, Machamer L, Asumbrado RC, Behrens MA (2018) Relationship between Nutrition (REAP), Exercise (VSAQ), and Stress on Premenstrual Syndrome Severity (PSST): Correlation, Cross-Section, Purposive Sample of 75 Females Ages 18-55. Physiother Rehabil 3: 158. doi: 10.4172/2573-0312.1000158

Page 5 of 6

premenstrual syndrome symptoms of women between the ages of 18 and 55 years. A linear regression analysis was run with predicting variables of VSAQ, REAP totals, stress level, BMI and REAP referral scores to determine a relationship between them and PSST diagnostic categories (no diagnosis, mod-severe, PMDD). A direct correlation between stress level and PSST diagnosis was found using a Spearman rho $(\mathrm{r}=0.324)(\mathrm{A}=75)(\mathrm{p}<0.005)$. Other variables were not found to be significant predictors for PSST diagnosis.

\section{Discussion}

\section{Major findings}

The major findings linked perceived stress levels directly and significantly with PMS severity of symptoms, with stress level being a significant predictor for outcomes of PSST $(\mathrm{r}=0.324)$ with a $(\mathrm{p}=0.005)$. Exercise intensity, frequency and duration, as well as nutritional intake were not significantly associated with PMS symptom severity in this study.

\section{Limitations}

A major limitation to the study was the homogeneity of the sample population. Over $50 \%$ of the women surveyed were between the ages of 18 22 years old. Though the sample showed high ethnic diversity, it diversity in BMI and exercise levels was limited. Healthy BMI, (18-24) was noted for greater than $60 \%$ of the women; only $5 \%$ had a BMI greater than 30 or overweight. Finally, $88 \%$ rated their level of exercise intensity as vigorous, with a VSAQ of 7 METs or greater. Specifically, $n=2$ for the low exercise intensity, $\mathrm{n}=7$ for the moderate and $\mathrm{n}=66$ for vigorous. The demographics of our study pulled a large group of individuals who exercised with a high intensity level. This is not representative of the lower exercise intensity level group, which may produce differing results.

\section{Implications}

Our study found the strongest relationship of PMS symptom severity was related to perceived stress level $(\mathrm{r}=0.324)$ with a $(\mathrm{p}<0.005)$. Furthermore, $20 \%(n=15 / 75)$ of the women reported and met the criteria by the PSST for moderate to severe PMS. Only one subject indicated no report of PMS symptoms. Meaning, $98.7 \%$ of women in the sample experienced some level of discomfort associated with PMS symptoms, enough to cause disruption in everyday living activities.

The results of the current study indicate there is no significant relationship between PMS symptom severity and exercise intensity or nutritional intake. However, the level of perceived stress was a significant indicator of PMS symptom severity and should be considered for future pharmacologic, biologic, behavioral and environmental determinants. The results of our study are in line with the Lustyk's research, who also found no relationship between exercise intensity and PMS symptoms [22]. However, our study specified exercise intensity and type as part of the factors of exercise thought to influence PMS symptom severity.

However, other research suggests exercise does have positive effects on PMS symptoms $[1,2,9,17]$. There is also evidence that moderate to high intensity exercise is more effective in reducing PMS symptoms as compared to low intensity exercise $[2,12]$. However, studies also support reduced PMS symptoms with consistent exercise, not dependent on intensity of exercise. Results from this research, between PMS symptoms and nutrition also showed no relationship, which contrasted with other literature. Bosarge article suggested lifestyle changes for decreasing symptoms of PMS including, but not limited to Vitamin B, calcium supplements and limiting sodium, caffeine and alcohol intake. Our results were limited in supplemental counts and we were unable to analyze the significance of $400 \mathrm{mg}$ Magnesium and Vitamin E. Many of the studies examined suggested that nutrition and intensity of exercise maybe correlated to reduce symptoms of PMS [2,12,16,23].

The results of our study with such high incidence amongst the sample of PMS symptoms and the link to perceived stress led us to consider this as important for rehabilitation related to chronic pain and function. Although a vast amount of women suffer from PMS symptoms, the multitude of symptoms, classifications and theories make diagnosing and treating difficult $[1-3,6,24]$. The various symptoms and reported intensity used for classifying make diagnosing difficult. Organizations such as WHO, NIHM, APA, and ACOG, offer differing guidelines for diagnosing PMS $[2,25]$. We feel that with the high number of women reporting symptoms further research needs to focus on determining a more uniformed diagnosis tool and treatment options for women.

\section{Further research suggestions}

In order to better serve women with PMS symptoms it is imperative that we address signs and symptoms early so they do not go through the difficulties that it brings with it. Our research indicated two large areas that need to be explored further:

1. Women who have PMS symptoms being overlooked and not diagnosed due to factors that can be standardized? Why were there $n=15 / 75$ women filling the questionnaire out who would of classified as PMS/PMDD according to their score but yet not diagnosed with PMS/ PMDD (per our prescreening to eliminate this group)?

2. How does perceived stress play a role in defining PMS symptom severity from a biological model? Environmental Model? Economic health administrative model? Employment model?

\section{Conclusion}

Our research found a high incidence of reported PMS symptoms and direct correlation of perceived stress levels with PMS symptom severity. Our research showed no significant relationship of PMS symptoms with factors of exercise or nutritional intake. Further research is needed to incorporate larger sample size for the low-intensity population in order to gain a more accurate depiction of the effects of exercise intensity on women suffering from PMS symptoms. Similarly, future research needs to include a larger sample size to account for the effects of supplemental Magnesium and Vitamin E. Perceived stress was the strongest prediction of PMS symptom severity. However, future research needs to further explore the effects of stress level on PMS symptom severity and related biomarkers including cortisol level fluctuations. Further examination of the criteria for the PSST should be examined to inform medical practice and health care professionals of the potential effects of limiting diagnostic criteria for intervention.

\section{References}

1. Daley (2009) Exercise and premenstrual symptomatology: a comprehensive review. J Womens Health 18: 895-899.

2. Vishnupriya R, Rajarajeswaram $P(2011)$ Effects of aerobic exercise at different intensities in pre menstrual syndrome. J O Gynecol India 61: 675-682.

3. Wakeman MP (2013) An open-label pilot study to assess the effectiveness of krill oil with added vitamins and phytonutrients in the relief of symptoms of PMS. Nutrit Dietar Supple 5

4. Hoyer J, Burmann I, Kieseler ML, Vollrath F, Hellrung L, et al. (2013) Menstrua cycle phase modulates emotional conflict processing in women with and without premenstrual syndrome (PMS)-a pilot study." PloS One 8: e59780.

5. Heinemann LAJ, Minh TD, Heinemann K, Lindemann M, Filonenko A (2012) Intercountry assessment of the impact of severe premenstrual disorders on 
Citation: Walton LM, Machamer L, Asumbrado RC, Behrens MA (2018) Relationship between Nutrition (REAP), Exercise (VSAQ), and Stress on Premenstrual Syndrome Severity (PSST): Correlation, Cross-Section, Purposive Sample of 75 Females Ages 18-55. Physiother Rehabil 3: 158. doi: 10.4172/2573-0312.1000158

work and daily activities. Healt care women internat 33: 109-124.

6. Freeman EW, Halberstadt SM, Rickels K, Legler JM, Lin H, et al. (2011) Core symptoms that discriminate premenstrual syndrome. J Womens Health 20: 29-35.

7. WHO (2007) International classification of functioning, disability, and health: children \& youth version: ICF-CY: World Health Organization.

8. Jehan S, Auguste E, Hussain M, Pandi-Perumal SR, Brzezinski A, et al. (2016) Sleep and Premenstrual Syndrome. J Sleep Med Disord 3: 1061.

9. Ann YK, O'Brien PM, Eriksson E (2008) Premenstrual syndrome. Lancet 371: 1200-1210.

10. ACOG Practice Bulletin (2000) Clinical management guidelines for obstetriciangynecologists. Number 15, April 2000. Premenstrual syndrome. Obstet Gynecol 95:1-9.

11. Anderson E, Shivakumar G (2013) Effects of exercise and physical activity on anxiety. Fronti Psych 4: 27.

12. Tonekaboni MM, Peeri M, Azarbayjani MA (2012) Effect of Two intensity of aerobic exercise on clinical symptoms of premenstrual syndrome in fertile women. World Appl Sci J 19: 295-301.

13. Bosarge PM (2003) Understanding and treating PMS/PMDD. Nursing 33: 13-14.

14. Chocano-Bedoya PO, Manson JE, Hankinson SE, Willett WC, Johnson SR (2011) Dietary B vitamin intake and incident premenstrual syndrome. Am J Clin Nutr 93: 1080-1086.

15. Filho EAR, Lima JC, Neto JSP, Montarroyos U (2011) Essential fatty acids for premenstrual syndrome and their effect on prolactin and total cholesterol levels: a randomized, double blind, placebo-controlled study. Reprod Health 8: 2.
16. Sohrabi N, Kashanian M, Ghafoori SS, Malakouti SK (2013) Evaluation of the effect of omega-3 fatty acids in the treatment of premenstrual syndrome: "A pilot trial". Complemen Therap Med 21: 141-146.

17. Steiner M, Macdougall M, Brown E (2003) The premenstrual symptoms screening tool (PSST) for clinicians. Archiv Wom Ment Healt 6: 203-209.

18. Raiten DJ, Ashour FAS, Ross AC, Meydani SN, Dawson HD, et al. (2015) Inflammation and nutrition science for programs/policies and interpretation of research evidence (INSPIRE). J Nutrit 145: 1039S-1108S.

19. Kraemer GR, Kraemer RR (1998) Premenstrual syndrome: diagnosis and treatment experiences. J Women Healt 7: 893-907.

20. Freeman EW, DeRubeis RJ, Rickels K (1996) Reliability and validity of a daily diary for premenstrual syndrome. Psych Res 65: 97-106.

21. Myers J, Bader D, Madhavan R, Froelicher V (2001) Validation of a specific activity questionnaire to estimate exercise tolerance in patients referred for exercise testing. Am Heart J 142: 1041-1046.

22. Lustyk MK, Widman L, Paschane A, Ecker E (2004) Stress, quality of life and physical activity in women with varying degrees of premenstrual symptomatology. Women \& health 39: 35-44.

23. Ghanbari Z, Manshavi FD, Jafarabadi M (2008) The effect of three months regular aerobic exercise on premenstrual syndrome. J Family Reproduct Healt 2: 167-171.

24. True BL, Goodner SM, Burns EA (1985) Review of the etiology and treatment of premenstrual syndrome. Drug Intelli Clinic Pharm 19: 714-722.

25. American Psychiatric Association (1994) Diagnostic and statistical manual of mental disorders. 